\title{
The Removal of the Quartic Potential in Gauge Theories and Asymptotic Freedom (Addendum).
}

J. P. HsU

Center for Particle Theory, The University of Texas at Austin - Austin, Tex.

(Lett. Nuovo Cimento, 12, $503(1975))$

There is an interesting possibility of removing the scalar field $\varphi_{3}$ from the theory (or the external states of a physical process) by adding a mass term $-m^{2} \varphi_{3}^{2} / 2$ to the effective Lagrangian $\mathscr{L}_{\text {eff }}=\mathscr{L}_{B}+\mathscr{L}_{\text {ff }}$ (cf. eqs. (10b), (11) and (12)) and taking the limit $m_{r}$ (renormalized $m$ ) $\rightarrow \infty$ eventually, similar to the spirit of the $\xi$-limiting for-

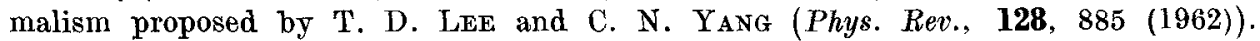
In this way, the resultant theory involves only the physical vector fields $\boldsymbol{B}_{\mu}$ : $\mathscr{L}_{\text {eff }}^{\prime}\left(\boldsymbol{B}_{\mu}\right)=\mathscr{L}_{\text {eff }}-\frac{1}{2}\left(m \varphi_{3}\right)^{2}$. The Lagrangian $\mathscr{L}_{\text {eff }}^{\prime}\left(\boldsymbol{B}_{\mu}\right)$ is also manifestly renormalizable by power counting. It leads to unitary amplitudes in the limit $m \rightarrow \infty$; this has been verified up to and including 2.loop diagrams (e.g. the processes in (13) and (14)). Also, it is interesting to note that the result (19) is in harmony with the result obtained by N. P. Chavg (Phys. Rev. D, 10, $2706(1974)$ ) in a related but different theory based on the eigenvalue-condition considerations. 